\title{
Primary ciliary dyskinesia: mechanisms and management
}

\author{
This article was published in the following Dove Press journal: \\ The Application of Clinical Genetics \\ 19 September 2017 \\ Number of times this article has been viewed
}

\section{Nadirah Damseh' \\ Nada Quercia',2 \\ Nisreen Rumman ${ }^{3}$ \\ Sharon D Dell ${ }^{4}$ \\ Raymond $\mathrm{H} \mathrm{Kim}^{5}$}

'Division of Clinical and Metabolic Genetics, ${ }^{2}$ Department of Molecular Genetics, University of Toronto, Toronto, ON, Canada; ${ }^{3}$ Pediatric Department, Makassed Hospital, Jerusalem, Palestine; ${ }^{4}$ Division of Respiratory Medicine, Department of Pediatrics, Child Health Evaluative Sciences, Hospital for Sick Children, ${ }^{5}$ Fred A Litwin Family Centre in Genetic Medicine, University Health Network and Mount Sinai Hospital, Department of Medicine, University of Toronto, Toronto, ON, Canada

Correspondence: Raymond H Kim Fred A Litwin Family Centre in Genetic Medicine, University Health Network, Mount Sinai Hospital, Department of Medicine, University of Toronto, Joseph and Wolf Lebovic Building, 60 Murray Street, Toronto, ON M5T 3L9, Canada $\mathrm{Tel}+\mathrm{I} 4165864800$ ext 4220

Fax +l 4166195523

Email raymond.kim@utoronto.ca
Abstract: Primary ciliary dyskinesia is a genetically heterogeneous disorder of motile cilia that is predominantly inherited in an autosomal-recessive fashion. It is associated with abnormal ciliary structure and/or function leading to chronic upper and lower respiratory tract infections, male infertility, and situs inversus. The estimated prevalence of primary ciliary dyskinesia is approximately one in 10,000-40,000 live births. Diagnosis depends on clinical presentation, nasal nitric oxide, high-speed video-microscopy analysis, transmission electron microscopy, genetic testing, and immunofluorescence. Here, we review its clinical features, diagnostic methods, molecular basis, and available therapies.

Keywords: genetic testing, Kartagener's syndrome, primary ciliary dyskinesia

\section{Introduction}

Primary ciliary dyskinesia (PCD) is a predominantly autosomal-recessive inherited disorder of mucociliary clearance secondary to ciliary dysfunction. The ciliary defect can be structural and/or functional, resulting in incompetent mucociliary clearance and mucus retention. It was first described clinically by Siewert in 1904 as a triad of chronic sinusitis, bronchiectasis, and dextrocardia. ${ }^{1} \mathrm{PCD}$ is associated with chronic upper and lower respiratory tract infections (otosinopulmonary disease), male infertility, and situs inversus. Respiratory distress in neonates, chronic suppurative lung disease, chronic serous otitis media, and chronic rhinosinusitis are the common respiratory presentations of PCD. ${ }^{2}$ Situs inversus is present in half of PCD cases. Some patients also have other organ laterality defects and heterotaxis (laterality defects combined with heart lesions). ${ }^{3}$ The estimated incidence of PCD is approximately one in 10,000-40,000 live births. ${ }^{4}$ In a European survey, the prevalence in European countries ranged from 1.3 to 111 diagnosed cases per million children aged 5-14 years. The median age at diagnosis was 5.3 years and 3.5 years in children with situs inversus. ${ }^{4}$ Additional surveys of patients with situs inversus and bronchiectasis in Norway and Japan estimated the incidence of PCD to be one per 10,000-20,000 births. ${ }^{5,6} \mathrm{PCD}$ is more common in certain ethnic groups with high rates of consanguinity, such as the Volendam population in the Netherlands, the British Asian population, and the Amish and Mennonite communities in the US. ${ }^{7-10}$

A diagnosis of PCD is confirmed by either biallelic mutations in a known PCD gene or a classic PCD ultrastructural ciliary defect observed by transmission electron microscopy (TEM). ${ }^{11,12}$ However, in up to $30 \%$ of suspected PCD cases, genetic testing and TEM can be nondiagnostic. Therefore, many ancillary tests may help to clarify 
the diagnosis, including nasal nitric oxide (nNO), high-speed videomicroscopy analysis (HSVA), and immunofluorescence (IF). Despite the presence of these advanced technologies, diagnosing PCD can be very challenging and is best done at an expert center. ${ }^{13}$ PCD may mimic other conditions that cause secondary ciliary dysfunction, like respiratory infections or airborne pollutant exposure (including cigarette smoke), and this may contribute to underdiagnosed or latediagnosed cases in practice. ${ }^{14}$ To date, there are 39 genes known to be associated with PCD. Two-thirds of PCD cases can be confirmed by identifying biallelic mutations in one of the PCD-causing genes. ${ }^{15}$ The most common PCD-causing mutations are in DNAH5 and DNAH11, which encode outer dynein arm (ODA) proteins. ${ }^{16}$

\section{Cilia structure}

Respiratory epithelial cells have approximately 200 cilia per cell that beat in a synchronized manner to clear respiratory secretions. Cilia consist of a ring of microtubule scaffolding called the axoneme, which is covered by the cell membrane and anchored to the cell by a basal body. Motile cilia found on the surface of the respiratory tract have nine peripheral microtubule pairs that surround a central microtubule pair; this gives rise to the " $9+2$ " arrangement of microtubules seen on cross-sections of an axoneme under electron microscopy. Each microtubule pair consists of one microtubule $A$ and one microtubule B. ODAs and inner DAs (IDAs) are present along the peripheral microtubules and contain ATPase, which allows the peripheral microtubular pairs to slide relatively to one another (Figure 1). There are three linkage systems to hold the axoneme together: the inner sheath to support the central microtubules, nexin proteins that join adjacent outer microtubules, and radial spokes that connect the central microtubules to the peripheral microtubule A. ${ }^{17}$

Mucociliary clearance is an essential host-defense mechanism for the entire respiratory system. It has two main compartments: mucus production by mucus-producing goblet cells, which traps inhaled pollutants and infectious debris, and effective ciliary movement of ciliated cells, which clears the mucus outside the airways. Compromised mucociliary clearance predisposes to chronic respiratory diseases. In PCD, abnormal ciliary ultrastructure and orientation result in ciliary dysfunction. ${ }^{18}$

Another type of motile cilia, called nodal cilia, have a "9+0" microtubule arrangement and are present in embryonic nodal plate cells. These cilia lack the central microtubule pair, leading to rotatory motion of the motile nodal cilia, which produces a leftward flow of fluid across the surface
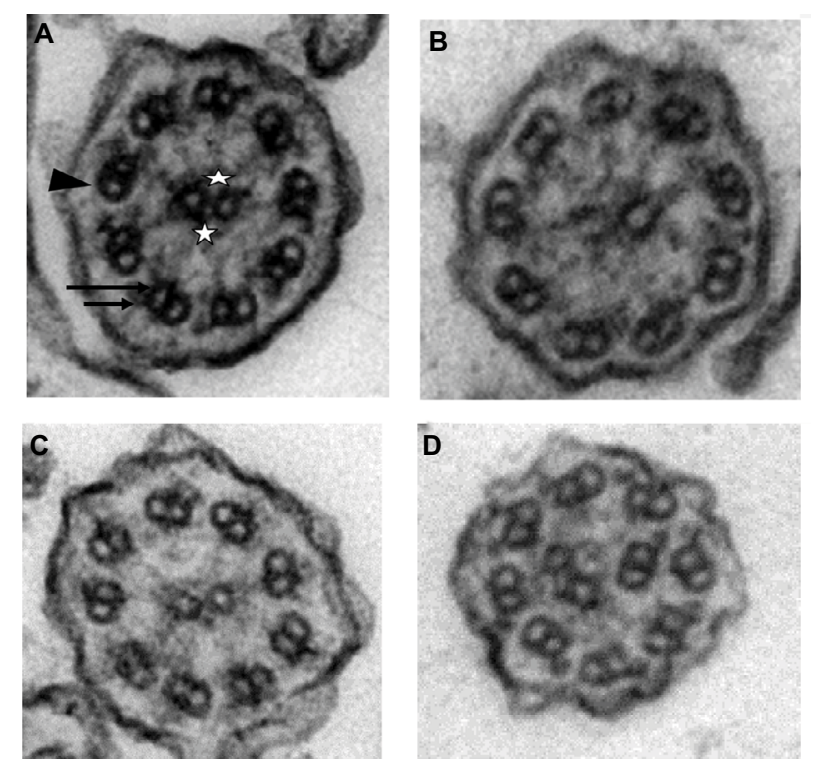

Figure I (A) Normal ciliary ultrastructures, as seen on transmission electron microscopy. This shows the motile cilia characterized a by typical " $9+2$ " arrangement with nine outer-microtubule doublets (arrowhead) and a central pair of microtubules (asterisk). The outer dynein arms (ODAs) and inner dynein arms (IDAs) (arrows) are present along the peripheral microtubules. (B) Absent ODAs. (C) Absent ODAs and IDAs. (D) Microtubular disorganization with IDAs. Note: Courtesy of Patricia Goggin and Regan Doherty from the Southampton PCD Centre, University Hospital NHS Trust, Southampton.

of the embryonic node. This nodal flow establishes left-right body orientation, and if interrupted it may create laterality defects such as situs inversus totalis, situs ambiguous, and heterotaxis syndromes. ${ }^{19}$ In PCD, organ orientation is a random event caused by dysfunctional nodal cilia responsible for laterality early in embryonic development, as evidenced by monozygotic female twins with PCD in which one twin has situs solitus and the other situs inversus totalis. ${ }^{20}$ More than 250 proteins are involved in axonemal structure and function, which means that mutations in genes encoding for any of these proteins may result in PCD. ${ }^{15}$

\section{Clinical presentation}

The clinical presentation of PCD is aspecific and overlaps with other common chronic respiratory diseases. However, there are some phenotypic clues that may lead physicians to early diagnosis of PCD and eventually early management and better prognosis.

Most PCD patients (73\%-91\%) present in the neonatal period with neonatal respiratory distress, which is commonly attributed to congenital pneumonia and transient respiratory distress (transient tachypnea of the newborn). ${ }^{21,22}$ Chronic nasal congestion is another aspecific presentation that is considered one of the hallmark features of PCD, especially if present at birth. ${ }^{2}$ 
In a case-control study of PCD patients with a history of neonatal respiratory distress, PCD cases required more and longer oxygen therapy, had later onset of neonatal respiratory distress (at 12 hours of age compared to 1 hour in control cases), and had higher frequency of lobar collapse (78\%) and situs inversus (48\%). The authors recommended a pediatric pulmonologist referral for a PCD assessment in patients with a combination of any two of lobar collapse on chest radiography, situs inversus, and oxygen therapy for $>2$ days (87\% sensitivity and $96 \%$ specificity for PCD). ${ }^{22}$

In early childhood, chronic upper and lower respiratory tract infections are the common presentation. In a cohort study of 118 PCD patients younger than 19 years from North America, clinical features included neonatal respiratory distress (82\%), chronic daily wet cough $(99 \%)$, chronic nasal congestion (97\%), and chronic or recurrent otitis media (92\%). ${ }^{2}$ Another American cohort study of 78 PCD patients from different age-groups showed high prevalence of chronic rhinosinusitis (100\%), recurrent otitis media ( $95 \%$ ), neonatal respiratory symptoms (73\%), and situs inversus (55\%). ${ }^{21}$

Adulthood PCD manifestations are similar to those in childhood. However, recurrent otitis media become less frequent at school age. Most men with PCD have infertility secondary to sperm immotility as a result of defective sperm-flagella movement. ${ }^{23,24}$ However, men with PCD due to mutations in the $C C D C 114$ gene have preserved fertility, partly due to lower CCDC114-transcript expression in testes compared to the respiratory system. ${ }^{10}$ Female fertility appears somewhat reduced, on account of egg transport relying at least partially on endometrial cilia. ${ }^{25}$

A large systemic review and meta-analysis was performed to quantify the variability in prevalence and severity of PCD clinical manifestations. A total of 52 studies were included, describing 1,970 patients of different age-groups. Except for the prevalence of congenital heart disease among PCD patients, the authors found considerable phenotypic heterogeneity in PCD manifestations between study cohorts. ${ }^{26}$ This illustrates the need for larger, concerted, prospective studies to identify the spectrum of PCD manifestations.

The European Respiratory Society guidelines for the diagnosis of PCD recommend an evaluation for PCD if several of the following clinical features exist: persistent wet cough, situs anomalies, congenital cardiac defects, persistent rhinitis, chronic middle-ear disease with or without hearing loss, history in term infants of neonatal upper and lower respiratory symptoms, or neonatal intensive care admittance. ${ }^{11} \mathrm{~A}$ recent consensus recommendation from the Genetic Disorders of Mucociliary Clearance Consortium in North America proposed PCD diagnostic criteria based on age (Table 1). ${ }^{12}$

Table I Recommended PCD diagnostic criteria by age

\section{Newborns (0-I month of age)}

Situs inversus totalis and unexplained neonatal respiratory distress at term birth plus at least one of the following:

- diagnostic ciliary ultrastructure on electron micrography

- biallelic mutations in one PCD-associated gene

- persistent and diagnostic ciliary waveform abnormalities on high-speed videomicroscopy, on multiple occasions

\section{Children (I month to 5 years)}

Two or more major PCD clinical criteria (see below) plus at least one of the following (nasal nitric oxide not included in this age-group, since it is not yet sufficiently tested):

- diagnostic ciliary ultrastructure on electron micrography

- biallelic mutations in one PCD-associated gene

- persistent and diagnostic ciliary waveform abnormalities on high-speed videomicroscopy, on multiple occasions

Children 5-18 years of age and adults

Two or more major PCD clinical criteria (see below) plus at least one of the following:

- nasal nitric oxide during plateau $<77 \mathrm{~nL} / \mathrm{min}$ on two occasions, $>2$ months apart, with cystic fibrosis excluded

- diagnostic ciliary ultrastructure on electron micrography

- biallelic mutations in one PCD-associated gene

- persistent and diagnostic ciliary waveform abnormalities on high-speed videomicroscopy, on multiple occasions

\section{Major clinical criteria for PCD diagnosis*}

1. Unexplained neonatal respiratory distress (at term birth) with lobar collapse and/or need for respiratory support with CPAP and/or oxygen for $>24$ hours.

2. Any organ-laterality defect: situs inversus totalis, situs ambiguous, or heterotaxis.

3. Daily, year-round wet cough starting in first year of life or bronchiectasis on chest CT.

4. Daily, year-round nasal congestion starting in first year of life or pansinusitis on sinus CT.

Notes: *Other diagnostic possibilities should have been considered, such as cystic fibrosis and immunodeficiencies, and diagnostic tests performed to rule out those disorders, as clinically indicated. Reproduced from Shapiro AJ, Zariwala MA, Ferkol T, et al. Diagnosis monitoring and treatment of primary ciliary dyskinesia: PCD Foundation consensus recommendations based on state of the art review. Pediatr Pulmonol. 2016;51:1 I5-132.12

Abbreviations: PCD, primary ciliary dyskinesia; CPAP, continuous positive airway pressure; CT, computed tomography. 


\section{Diagnostic testing}

The clinical and genetic heterogeneity of PCD contribute to the challenges of diagnosis. The diagnosis of PCD is still difficult, despite the availability of sophisticated diagnostic tests. Currently, diagnosis incorporates multiple complex and expensive technologies, including nNO, HSVA, TEM, genetic testing, and IF of ciliary proteins. The diagnosis of PCD can be confirmed by genetic testing (biallelic mutations in known PCD genes) and/or finding a classic ultrastructural defect on TEM. However, if these diagnostic tests are not accessible or results are inconclusive, other investigations, such as nNO and HSVA, can play an essential role in making the diagnosis of PCD more likely, aiding in the management of putative PCD patients.

$\mathrm{nNO}$ is low in patients with PCD $(10 \%-15 \%$ of normal values). There is no clear explanation for this, but some authors have suggested that impaired NO synthase 2 function may have a significant contribution. ${ }^{27}$ The $\mathrm{nNO}$ test is a reliable PCD-screening test performed by measuring NO during a velum-closure maneuver, with good sensitivity and specificity in cooperative children ( $>5$ years old) and adults. $\mathrm{nNO}$ can also be measured during tidal breathing in less cooperative children ( $<5$ years old), but with lower sensitivity. ${ }^{28}$ Sporadic cases of PCD have been reported, with normal nNO suggesting it is not $100 \%$ sensitive for $\mathrm{PCD}^{27}$ Cystic fibrosis (CF) and pulmonary hypertension are other conditions known to have low $\mathrm{nNO}$ levels, suggesting that $\mathrm{nNO}$ is not $100 \%$ specific for PCD. ${ }^{29}$ A prospective North American study defined a disease-specific nNO-cutoff value of $77 \mathrm{~nL} /$ min, with sensitivity of $98 \%$ and specificity of $99.9 \% .^{30}$

HSVA is a subjective and qualitative analysis of ciliary beat pattern and frequency of ciliated epithelium-biopsy samples (eg, nasal or bronchoscopic brush). PCD and non-PCD patients show a degree of functional ciliary abnormalities in HSVA without culture. However, after air-liquid interface culture, normal ciliary beat pattern was seen in all non-PCD patients and was uniformly abnormal in PCD patients. ${ }^{31}$ The test has excellent sensitivity (93\%) and specificity (93\%), and many European centers consider it a first-line test in the diagnostic algorithm of $\mathrm{PCD} .{ }^{32}$ However, HSVA requires great expertise to differentiate between primary and secondary abnormalities. In addition, some PCD mutations have been reported with subtle beating abnormalities, eg, GAS 8 mutations with radial spoke defects. ${ }^{33}$ This raises the concern that despite high sensitivity and specificity, HSVA cannot be used as a stand-alone test. ${ }^{32}$

TEM was once considered the "gold standard" for the diagnosis of PCD. This has changed, since a substantial number of PCD cases are caused by mutations that are not associated with ultrastructural defects, eg, DNAH11 and nexin-link defects (CCDC65, CCDC164), and thus would not be identified by TEM. ${ }^{34,35}$ While the detection rate of TEM is $83 \%$, TEM has been estimated to miss the diagnosis of PCD in $26 \%$ of cases. Therefore, normal TEM does not rule out PCD. The prevalence of ultrastructural defects detected by TEM is $28 \%$ for ODA defect, $26 \%$ for both ODA and IDA defects, and $10 \%$ for microtubular defects. ${ }^{36}$

Genetic studies can provide a definitive diagnosis of PCD if biallelic mutations are identified in an autosomal-recessive PCD-causing gene or a hemizygous mutation is identified in an X-linked gene. However, genetic testing does not substitute other PCD functional studies, because in up to $35 \%$ of PCD cases, the genes are not yet known. So far, 39 PCD-causing genes have been identified. It is postulated that any of the $>200$ genes involved in ciliogenesis could contribute to PCD. Until now, only $65 \%$ of PCD patients have had a genetically confirmed diagnosis. ${ }^{37}$

IF is a form of indirect imaging that labels ciliary proteins using IF-linked antibodies and fluorescent or confocal microscopy. It helps in understanding the effect of genetic mutations on ciliary proteins. Using different fluorescence tags may provide further information regarding protein localization. IF is able to detect all ultrastructural abnormalities identifiable by TEM, in addition to abnormalities of nexin-link components. ${ }^{38}$ Some authors support including IF in the routine diagnostic approach, especially if TEM equipment or expertise is not available. ${ }^{39}$

The European Respiratory Society proposed a PCDdiagnosis algorithm of three steps: 1) nNO and HSVA, 2) TEM, and 3) targeted genetic testing based on the presence or absence of TEM defects, and repeat HSVA \pm cell culture. ${ }^{11}$ Abnormalities seen on HSVA can be secondary to damage during sampling and local infection or inflammation; therefore, a repeat sample after treating the patient might be needed. Reanalysis of cilia following culture and redifferentiation of epithelial cells at an air-liquid interface helps to eliminate these secondary abnormalities, hence aiding in the diagnosis. ${ }^{31}$ With the decreasing costs of genetic sequencing and advances in massively parallel sequencing, genetic testing could emerge as a diagnostic alternative in centers that lack many of the other diagnostic investigations. ${ }^{40}$

\section{Genetics \\ PCD genes and inheritance}

PCD is primarily inherited in an autosomal-recessive fashion, and to date 39 PCD-associated genes have been identified. Around $65 \%$ of PCD patients have been identified to have biallelic mutations in one of these genes, and the majority 
of PCD mutations have loss-of-function variants, including nonsense, frameshift, and splice-site mutations. ${ }^{15}$ Recently, a study has demonstrated X-linked inheritance associated with point mutations and large deletions in PIH1D3, a cytoplasmic protein involved in dynein assembly. ${ }^{41}$

DNAII was the first gene identified to be associated with PCD in 1999 through orthologue studies. Chlamydomonas reinhardtii is a single-cell alga with two flagella containing an axonemal structure similar to that of human respiratory cilia and sperm tails. Mutant $C$. reinhardtii carrying a defect in $I C 78$, a gene that encodes the intermediate chain of dynein, has been found to have axonemal ultrastructural defects (absence of ODAs), similar to some patients with PCD. The human orthologue DNAII was subsequently confirmed to be associated with PCD through a candidate-gene approach. ${ }^{42}$ In addition to DNAII, the most common identified causative genes of PCD include DNAH5, DNAH11, CCDC39, CCDC40, DNAAF1, LRRC6, and DNAI2 (Table 2). ${ }^{15,43-45}$ Other PCD genes include ZMYND10, TTC25, SPAG1, RSPH9, RSPH4A, RSPH3, RSPH1, TXNDC3 (NME8), HYDIN, HEATR2, GAS8, DYX1C1, CCDC164 (DRC1), DNAL1, DNAJB13, DNAAF2 (KTU), DNAAF3 (C19orf51), CCNO, CCDC65(DRC2), CCDC151, CCDC114, CCDC103, C21orf59, ARMC4, STK36, DNAH6, DNAH8, MCIDAS, RPGR, and OFD1. ${ }^{46-50}$

Ciliary ultrastructural defects are identified in the majority of PCD patients (70\%). These include ultrastructural defects of ODA defects, IDA defects, central microtubular pair abnormalities and radial spoke defects. However, some PCD patients have normal ciliary ultrastructure, mainly those with DNAH11, HYDIN, CCDC164, and CCDC65 mutations. ${ }^{34,44,53,55,56}$

\section{Genotype-phenotype correlation}

Though limited, some genotype-phenotype correlations have been identified. For example, a previous study found mutations in $R P G R$ in patients with a complex X-linked phenotype of PCD and retinitis pigmentosa. ${ }^{57}$ Another study reported a large family with $\mathrm{X}$-linked recessive mental retardation syndrome. They presented with macrocephaly and ciliary dysfunction as a result of a frameshift mutation in the $O F D 1$ gene. ${ }^{58}$

$C C D C 39$ and $C C D C 40$ mutations are characterized by IDA defects and microtubular disorganization. Mutations in either gene lead to an indistinguishable clinical phenotype and ciliary defects. They are associated with earlier presentation, worse lung function, structural changes, and poorer nutritional status. ${ }^{2}$ Mutations in RSHP1 are associated with a milder disease phenotype, higher levels of nNO, and better lung function. ${ }^{59}$ Mutations in $C C N O$ lead to paucity or complete absence of motile cilia. Affected patients have earlyonset and progressive PCD symptoms; situs inversus has not been not observed, and few have infertility. ${ }^{60} C C D C 114$ mutations have no significant effect on fertility, and this has been attributed mainly to low transcript expression of $C C D C 114$ in testes compared to respiratory ciliated cells. ${ }^{10}$

Nodal cilia determine left-right asymmetry. They contain a 9+0 axonemal composition, and lack the components of central apparatus and radial spokes. Therefore, patients with mutations in genes that encode the central complex and radial spokes (eg, RSPH1, RSPH3, RSPH4A, RSPH9, and HYDIN) do not have situs abnormalities, as they still have the normal rotatory function, which is important for normal left-right asymmetry. ${ }^{55,61}$

\section{Genetic testing}

PCD is a genetically heterogeneous disease. Traditionally, genes were sequentially analyzed using Sanger sequencing based on ciliary ultrastructure and the most prevalent PCD genes. The advent of next-generation sequencing and decreasing costs have led to the development of a number

Table 2 Common genes associated with autosomal recessive PCD

\begin{tabular}{|c|c|c|c|c|}
\hline Gene name & $\begin{array}{l}\text { Percentage } \\
\text { of all PCD }\end{array}$ & $\begin{array}{l}\text { Ciliary ultrastructural } \\
\text { defect }\end{array}$ & Percentage based on ultrastructural defect & References \\
\hline DNAH5 & $15 \%-21 \%$ & ODA defect & $27 \%-38 \%$ of PCD with ODA defects & $|5,5|, 52$ \\
\hline DNAHI I & $6 \%-9 \%$ & Normal ultrastructure & $48 \%$ of PCD with normal ultrastructure & $|5,5|-53$ \\
\hline CCDC39 & $2 \%-10 \%$ & $\begin{array}{l}\text { IDA defect and microtubular } \\
\text { disorganization }\end{array}$ & $\begin{array}{l}36 \%-65 \% \text { of PCD with IDA defect and microtubular } \\
\text { disorganization }\end{array}$ & 15,52 \\
\hline DNAII & $2 \%-10 \%$ & ODA defect & $4 \%-13 \%$ of PCD with ODA defects & $15,16,52$ \\
\hline CCDC40 & $2 \%-8 \%$ & $\begin{array}{l}\text { IDA defect and microtubular } \\
\text { disorganization }\end{array}$ & $\begin{array}{l}24 \%-54 \% \text { of PCD with IDA defect and microtubular } \\
\text { disorganization }\end{array}$ & $15,52,54$ \\
\hline DNAAFI (LRRC50) & $4 \%-5 \%$ & ODA and IDA defects & $17 \%$ of PCD with both ODA and IDA defects & 15,52 \\
\hline LRRC6 & $3 \%$ & ODA and IDA defects & II\% of PCD with both ODA and IDA defects & 15 \\
\hline DNAI2 & $2 \%$ & ODA defect & $4 \%$ of PCD with ODA defects & 15,52 \\
\hline
\end{tabular}

Note: Other PCD genes include ZMYNDI0, TTC25, SPAGI, RSPH9, RSPH4A, RSPH3, RSPHI, PIHID3, TXNDC3 (NME8), HYDIN, HEATR2, GAS8, DYXICI, CCDCI64 (DRCI), DNALI, DNAJBI3, DNAAF2 (KTU), DNAAF3 (CI9orf5I), CCNO, CCDC65 (DRC2), CCDCI5I, CCDCII 4, CCDCI03, C2 I orf59, ARMC4, STK36, DNAH6, DNAH8, MCIDAS, RPGR, and OFDI, in addition to two linkage regions: CILD4 and CILD8.

Abbreviations: PCD, primary ciliary dyskinesia; ODA, outer dynein arm; IDA, inner DA. 
of gene panels based on massively parallel sequencing. However, the pace of PCD-gene discovery is quickly outdating currently available gene panels, suggesting alternative molecular genetic approaches may be needed. ${ }^{44}$ It is likely that many more PCD-causing genes will be discovered, given the hundreds of genes that encode proteins for cilia structure and function.

The use of whole-exome sequencing (WES) and wholegenome sequencing (WGS) plays a significant role in novelgene discovery in genetically heterogeneous diseases, such as PCD. ${ }^{62}$ WES and WGS provide the advantage of analyzing all potential causative genes, including recently discovered genes that might not be included on PCD-gene panels. ${ }^{63}$ As such, it has been suggested that WES could be more costand time-effective as a first-line molecular diagnostic test for PCD than clinical gene panels. ${ }^{13,64}$ One limitation of WES is copy-number variant analysis, which can be a significant proportion of PCD mutations. ${ }^{62}$ This may be overcome with more refined copy-number variant algorithms or with WGS. ${ }^{63}$ WGS specifically analyzes noncoding regions that might be responsible for decreased expression of PCD genes, and could be used in conjunction with ciliary IF to correlated novel noncoding variants.

\section{Management}

Proposed therapies for PCD lack randomized controlled trials, and as such are not evidence-based but mainly extrapolated from the field of CF. The aim of PCD treatment is to maintain or recover lung function by early detection and aggressive management of complications. The main objectives are the clearance of mucus, prevention of respiratory infections, and vigorous treatment of bacterial infections.

Measures used to enhance mucus clearance include positive pressure expiratory devices, intrathoracic oscillatory devices, high-frequency chest compression using vest therapy, manual chest physiotherapy, postural drainage, autogenic drainage, active cycle breathing, and exercise. ${ }^{65}$ One study in children reported that physical exercise before physiotherapy is a more potent stimulus for bronchodilation than inhaled $\beta_{2}$-agonists. ${ }^{66}$ Exercise may promote deep breathing and cough, which helps in mucus clearance. There is very limited evidence for using inhaled medications in PCD. A recent randomized clinical trial showed little evidence of quality-of-life improvement with the use of inhaled hypertonic saline in PCD patients. ${ }^{67}$

Prophylactic courses of antibiotics are used in some centers, but this is not routinely recommended. To address this, a multicenter European clinical trial is under way to assess the efficacy and safety of azithromycin as maintenance therapy for 6 months in PCD patients. Azithromycin is known to have antibacterial, anti-inflammatory, and anti-quorum-sensing properties and is commonly used in chronic respiratory diseases, such as $\mathrm{CF}^{68}$

The cornerstone of PCD management is the prompt treatment of respiratory tract infections. Management should be guided by microbiological studies using sputum culture, cough-swab culture, and sometimes bronchoalveolar lavage. ${ }^{38}$ Commonly isolated pathogens from sputum culture in PCD patients are Haemophilus influenzae (most common, 65\%), Streptococcus pneumoniae, Staphylococcus aureus, Moraxella catarrhalis, Pseudomonas aeruginosa, and mucoid P. aeruginosa. ${ }^{68}$

Bronchiectasis is a prominent feature in PCD, and surgical resection with lobectomy or segmentectomy in patients with bronchiectasis is thought to decrease the risk of infection progressing into healthier lung tissue. However, there is controversy in the literature regarding the benefit of lung resection in PCD patients. PCD patients with severe localized bronchiectasis have been offered lobectomy where failed medical management has caused significant morbidity. In one cohort, $85 \%$ (eleven of 13) of lobectomized PCD patients reported subjective symptomatic improvement. ${ }^{69}$ However, lobectomized PCD patients had the same prevalence of respiratory symptoms as the unlobectomized patients. Another cohort study showed that lobectomized patients had significantly worse forced expiratory volume in one second and forced vital capacity compared to unlobectomized patients. The authors considered lobectomy a poor prognostic factor for PCD in adults. ${ }^{69,70}$

A recent in vitro study demonstrated the efficacy of premature termination codon (PTC) read-through stimulation in PCD-causing mutations with aminoglycosides. PTC readthrough has been shown to restore functional protein expression and reduce symptoms in several genetic disorders caused by loss-of-function mutations. In this study, three types of aminoglycosides were used to examine its effect on five PCD genes with PTC: DNAH5, DNAH11, CCDC40, RSPH4A, and $S P A G 1$. The efficacy of each aminoglycoside in stimulating PTC read-through and protein production depended on the particular PTC, as well as on drug type and concentration. Among the examined PTCs, suppression was most efficient for the stop codon UGA. This precision medicine for PCD does show promise, but further studies are required to validate its efficacy, as it was a preclinical cell-line study. ${ }^{71}$

Lung transplantation has been described in a few case reports, and indicated increased 1-year mortality. More recently, an American study using a national registry 
demonstrated that the survival outcome of a large group of patients with PCD and Kartagener's syndrome was similar to that of the general population with lung transplantation for common indications like COPD and $\mathrm{CF}^{72}$

\section{Conclusion}

PCD is a genetically heterogeneous recessive condition with defective ciliary motility. It is characterized by otosinopulmonary disease, male infertility, and situs inversus. A diagnosis of PCD depends on clinical presentation, nNO, HSVA, TEM, and genetic testing. The rapid development in PCD genetics is promising. In the near future, genetic testing could become the main diagnostic method, with minimal use of other expensive and effort-demanding diagnostic modalities. Due to its rarity and lack of clinical trials, the management of PCD remains challenging and mainly supportive. Understanding the biology of PCD with respect to ciliary function may allow for more targeted therapeutic agents in future.

\section{Disclosure}

The authors report no conflicts of interest in this work.

\section{References}

1. Siewert A. Uber einen Fall von Bronchiectasie bei einem Patienten mit situs inversus viscerum. Berl Klin Wochenschr. 1904;41:139-141.

2. Davis SD, Ferkol TW, Rosenfeld M, et al. Clinical features of childhood primary ciliary dyskinesia by genotype and ultrastructural phenotype. Am J Respir Crit Care Med. 2015;191:316-324.

3. Shapiro AJ, Davis SD, Ferkol T, et al. Laterality defects other than situs inversus totalis in primary ciliary dyskinesia: insights into situs ambiguus and heterotaxy. Chest. 2014;146:1176-1186.

4. Kuehni CE, Frischer T, Strippoli MP, et al. Factors influencing age at diagnosis of primary ciliary dyskinesia in European children. Eur Respir J. 2010;36:1248-1258

5. Katsuhara K, Kawamoto S, Wakabayashi T, Belsky JL. Situs inversus totalis and Kartagener's syndrome in a Japanese population. Chest. 1972;61:56-61.

6. Torgersen J. Transposition of viscera - bronchiectasis and nasal polyps: a genetical analysis and a contribution to the problem of constitution. Acta Radiol. 1947;28:17-24.

7. Ferkol TW, Puffenberger EG, Lie H, et al. Primary ciliary dyskinesiacausing mutations in Amish and Mennonite communities. J Pediatr. 2013;163:383-387.

8. Lie H, Zariwala MA, Helms C, et al. Primary ciliary dyskinesia in Amish communities. J Pediatr. 2010;156:1023-1025.

9. O'Callaghan C, Chetcuti P, Moya E. High prevalence of primary ciliary dyskinesia in a British Asian population. Arch Dis Child. 2010;95: $51-52$.

10. Onoufriadis A, Paff T, Antony D, et al. Splice-site mutations in the axonemal outer dynein arm docking complex gene CCDC114 cause primary ciliary dyskinesia. Am J Hum Genet. 2013;92:88-98.

11. Lucas JS, Barbato A, Collins SA. European Respiratory Society guidelines for the diagnosis of primary ciliary dyskinesia. Eur Respir J. 2017;49:1601090.

12. Shapiro AJ, Zariwala MA, Ferkol T, et al. Diagnosis monitoring and treatment of primary ciliary dyskinesia: PCD Foundation consensus recommendations based on state of the art review. Pediatr Pulmonol. 2016;51:115-132.
13. Lucas JS, Leigh MW. Diagnosis of primary ciliary dyskinesia: searching for a gold standard. Eur Respir J. 2014;44:1418-1422.

14. Tilley AE, Walters MS, Shaykhiev R, Crystal RG. Cilia dysfunction in lung disease. Annu Rev Physiol. 2015;77:379-406.

15. Knowles MR, Daniels LA, Davis SD, Zariwala MA, Leigh MW. Primary ciliary dyskinesia: recent advances in diagnostics genetics and characterization of clinical disease. Am J Respir Crit Care Med. 2013;188:913-922.

16. Zariwala MA, Leigh MW, Ceppa F, et al. Mutations of DNAI1 in primary ciliary dyskinesia: evidence of founder effect in a common mutation. Am J Respir Crit Care Med. 2006;174:858-866.

17. Lodish H, Berk A, Zipursky SL, Matsudaira P, Baltimore D, Darnell J. Molecular Cell Biology. 4th ed. New York: WH Freeman; 2000.

18. Antunes MB, Cohen NA. Mucociliary clearance: a critical upper airway host defense mechanism and methods of assessment. Curr Opin Allergy Clin Immunol. 2007;7:5-10.

19. Hirokawa N, Tanaka Y, Okada Y, Takeda S. Nodal flow and the generation of left-right asymmetry. Cell. 2006;125:33-45.

20. Noone PG, Bali D, Carson JL, et al. Discordant organ laterality in monozygotic twins with primary ciliary dyskinesia. Am J Med Genet. 1999;82:155-160.

21. Noone PG, Leigh MW, Sannuti A, et al. Primary ciliary dyskinesia: diagnostic and phenotypic features. Am J Respir Crit Care Med. 2004;169: 459-467.

22. Mullowney T, Manson D, Kim R, Stephens D, Shah V, Dell S. Primary ciliary dyskinesia and neonatal respiratory distress. Pediatrics. 2014;134:1160-1166.

23. Munro NC, Currie DC, Lindsay KS, et al. Fertility in men with primary ciliary dyskinesia presenting with respiratory infection. Thorax. 1994;49:684-687.

24. Bush A, Chodhari R, Collins N, et al. Primary ciliary dyskinesia: current state of the art. Arch Dis Child. 2007;92:1136-1140.

25. Afzelius BA. Cilia-related diseases. J Pathol. 2004;204:470-477.

26. Goutaki M, Meier AB, Halbeisen FS, et al. Clinical manifestations in primary ciliary dyskinesia: systematic review and meta-analysis. Eur Respir J. 2016;48:1081-1095.

27. Walker WT, Jackson CL, Lackie PM, Hogg C, Lucas JS. Nitric oxide in primary ciliary dyskinesia. Eur Respir J. 2012;40:1024-1032.

28. Beydon N, Chambellan A, Alberti C, et al. Technical and practical issues for tidal breathing measurements of nasal nitric oxide in children. Pediatr Pulmonol. 2015;50:1374-1382.

29. Abba AA. Exhaled nitric oxide in diagnosis and management of respiratory diseases. Ann Thorac Med. 2009;4:173-181.

30. Leigh MW, Hazucha MJ, Chawla KK, et al. Standardizing nasal nitric oxide measurement as a test for primary ciliary dyskinesia. Ann Am Thorac Soc. 2013;10:574-581.

31. Hirst RA, Jackson CL, Coles JL, et al. Culture of primary ciliary dyskinesia epithelial cells at air-liquid interface can alter ciliary phenotype but remains a robust and informative diagnostic aid. PloS One. 2014;9:e89675.

32. Jackson CL, Behan L, Collins SA, et al. Accuracy of diagnostic testing in primary ciliary dyskinesia. Eur Respir J. 2016;47:837-848.

33. Frommer A, Hjeij R, Loges NT, et al. Immunofluorescence analysis and diagnosis of primary ciliary dyskinesia with radial spoke defects. Am J Respir Cell Mol Biol. 2015;53:563-573.

34. Horani A, Brody SL, Ferkol TW, et al. CCDC65 mutation causes primary ciliary dyskinesia with normal ultrastructure and hyperkinetic cilia. PloS One. 2013;8:e72299.

35. Schwabe GC, Hoffmann K, Loges NT, et al. Primary ciliary dyskinesia associated with normal axoneme ultrastructure is caused by DNAH11 mutations. Hum Mutat. 2008;29:289-298.

36. Kouis P, Yiallouros PK, Middleton N, Evans JS, Kyriacou K, Papatheodorou SI. Prevalence of primary ciliary dyskinesia in consecutive referrals of suspect cases and the transmission electron microscopy detection rate: a systematic review and meta-analysis. Pediatr Res. 2017;81:398-405,

37. Kurkowiak M, Ziętkiewicz E, Witt M. Recent advances in primary ciliary dyskinesia genetics. J Med Genet. 2015;52:1-9. 
38. Werner C, Onnebrink JG, Omran H. Diagnosis and management of primary ciliary dyskinesia. Cilia. 2015;4:2.

39. Shoemark A, Frost E, Dixon M, et al. Accuracy of immunofluorescence in the diagnosis of primary ciliary dyskinesia. Am J Respir Crit Care Med. 2017;196:94-101.

40. Rumman N, Jackson C, Collins S, Goggin P, Coles J, Lucas JS. Diagnosis of primary ciliary dyskinesia: potential options for resource-limited countries. Eur Respir Rev. 2017;26:160058.

41. Olcese C, Patel MP, Shoemark A, et al. X-linked primary ciliary dyskinesia due to mutations in the cytoplasmic axonemal dynein assembly factor PIH1D3. Nat Commun. 2017;8:14279.

42. Pennarun G, Escudier E, Chapelin C, et al. Loss-of-function mutations in a human gene related to Chlamydomonas reinhardtii dynein IC78 result in primary ciliary dyskinesia. Am J Hum Genet. 1999;65:1508-1519.

43. Kano G, Tsujii H, Takeuchi K, et al. Whole-exome sequencing identification of novel DNAH5 mutations in a young patient with primary ciliary dyskinesia. Mol Med Rep. 2016;14:5077-5083.

44. Kim RH, Hall DA, Cutz E, et al. The role of molecular genetic analysis in the diagnosis of primary ciliary dyskinesia. Ann Am Thorac Soc. 2014;11:351-359.

45. Dehlink E, Hogg C, Carr SB, Bush A. Clinical phenotype and current diagnostic criteria for primary ciliary dyskinesia. Expert Rev Respir Med. Epub 2016 Oct 19.

46. Hjeij R, Lindstrand A, Francis R, et al. ARMC4 mutations cause primary ciliary dyskinesia with randomization of left/right body asymmetry. $\mathrm{Am}$ J Hum Genet. 2013;93:357-367.

47. Knowles MR, Ostrowski LE, Loges NT, et al. Mutations in SPAG1 cause primary ciliary dyskinesia associated with defective outer and inner dynein arms. Am J Hum Genet. 2013;93:711-720.

48. Loges NT, Olbrich H, Becker-Heck A, et al. Deletions and point mutations of LRRC50 cause primary ciliary dyskinesia due to dynein arm defects. Am J Hum Genet. 2009;85:883-889.

49. Moore DJ, Onoufriadis A, Shoemark A, et al. Mutations in ZMYND10, a gene essential for proper axonemal assembly of inner and outer dynein arms in humans and flies cause primary ciliary dyskinesia. Am J Hum Genet. 2013;93:346-356.

50. Tarkar A, Loges NT, Slagle CE, et al. DYX1C1 is required for axonemal dynein assembly and ciliary motility. Nat Genet. 2013;45:995-1003.

51. Zariwala MA, Knowles MR, Omran H. Genetic defects in ciliary structure and function. Annu Rev Physiol. 2007;69:423-450.

52. Zariwala MA, Knowles MR, Leigh MW. Primary ciliary dyskinesia. 1993. Available from: https://www.ncbi.nlm.nih.gov/books/NBK1122. Accessed August 18, 2017.

53. Knowles MR, Leigh MW, Carson JL, et al. Mutations of DNAH11 in patients with primary ciliary dyskinesia with normal ciliary ultrastructure. Thorax. 2012;67:433-441.

54. Antony D, Becker-Heck A, Zariwala MA, et al. Mutations in CCDC39 and CCDC40 are the major cause of primary ciliary dyskinesia with axonemal disorganization and absent inner dynein arms. Hum Mutat. 2013;34:462-472.

55. Olbrich H, Schmidts M, Werner C, et al. Recessive HYDIN mutations cause primary ciliary dyskinesia without randomization of left-right body asymmetry. Am J Hum Genet. 2012;91:672-684.

56. Wirschell $\mathrm{M}$, Olbrich $\mathrm{H}$, Werner $\mathrm{C}$, et al. The nexin-dynein regulatory complex subunit DRC1 is essential for motile cilia function in algae and humans. Nat Genet. 2013;45:262-268.

The Application of Clinical Genetics

\section{Publish your work in this journal}

The Application of Clinical Genetics is an international, peer-reviewed open access journal that welcomes laboratory and clinical findings in the field of human genetics. Specific topics include: Population genetics; Functional genetics; Natural history of genetic disease; Management of genetic disease; Mechanisms of genetic disease; Counselling and ethical
57. Moore A, Escudier E, Roger G, et al. RPGR is mutated in patients with a complex $\mathrm{X}$ linked phenotype combining primary ciliary dyskinesia and retinitis pigmentosa. J Med Genet. 2006;43:326-333.

58. Budny B, Chen W, Omran H, et al. A novel X-linked recessive mental retardation syndrome comprising macrocephaly and ciliary dysfunction is allelic to oral-facial-digital type I syndrome. Hum Genet. 2006;120: $171-178$.

59. Knowles MR, Ostrowski LE, Leigh MW, et al. Mutations in RSPH1 cause primary ciliary dyskinesia with a unique clinical and ciliary phenotype. Am J Respir Crit Care Med. 2014;189:707-717.

60. Wallmeier J, Al-Mutairi DA, Chen CT, et al. Mutations in CCNO result in congenital mucociliary clearance disorder with reduced generation of multiple motile cilia. Nat Genet. 2014;46:646-651.

61. Jeanson L, Copin B, Papon JF, et al. RSPH3 mutations cause primary ciliary dyskinesia with central-complex defects and a near absence of radial spokes. Am J Hum Genet. 2015;97:153-162.

62. Neveling K, Feenstra I, Gilissen C, et al. A post-hoc comparison of the utility of Sanger sequencing and exome sequencing for the diagnosis of heterogeneous diseases. Hum Mutat. 2013;34:1721-1726.

63. Boycott K, Hartley T, Adam S, et al. The clinical application of genome-wide sequencing for monogenic diseases in Canada: position statement of the Canadian College of Medical Geneticists. J Med Genet. 2015;52:431-437.

64. Marshall CR, Scherer SW, Zariwala MA, et al. Whole-exome sequencing and targeted copy number analysis in primary ciliary dyskinesia. G3 (Bethesda). 2015;5:1775-1781.

65. Daniels ML, Noone PG. Genetics diagnosis and future treatment strategies for primary ciliary dyskinesia. Expert Opin Orphan Drugs. 2015;3:31-44.

66. Phillips GE, Thomas S, Heather S, Bush A. Airway response of children with primary ciliary dyskinesia to exercise and $\beta_{2}$-agonist challenge. Eur Respir J. 1998;11:1389-1391.

67. PaffT, Daniels JM, Weersink EJ, Lutter R, Noordegraaf AV, Haarman EG. A randomised controlled trial on the effect of inhaled hypertonic saline on quality of life in primary ciliary dyskinesia. Eur Respir J. 2017;49: 1601770 .

68. Kobbernagel HE, Buchvald FF, Haarman EG, et al. Study protocol rationale and recruitment in a European multi-centre randomized controlled trial to determine the efficacy and safety of azithromycin maintenance therapy for 6 months in primary ciliary dyskinesia. $B M C$ Pulm Med. 2016;16:104.

69. Smit HJ, Schreurs AJ, Van den Bosch JM, Westermann CJ. Is resection of bronchiectasis beneficial in patients with primary ciliary dyskinesia? Chest. 1996;109:1541-1544.

70. Yiallouros PK, Kouis P, Middleton N, et al. Clinical features of primary ciliary dyskinesia in Cyprus with emphasis on lobectomized patients. Respir Med. 2015;109:347-356.

71. Bukowy-Bieryllo Z, Dabrowski M, Witt M, Zietkiewicz E. Aminoglycoside-stimulated readthrough of premature termination codons in selected genes involved in primary ciliary dyskinesia. RNA Biol. 2016;13: 1041-1050.

72. Hayes D Jr, Reynolds SD, Tumin D. Outcomes of lung transplantation for primary ciliary dyskinesia and Kartagener syndrome. J Heart Lung Transplant. 2016;35:1377-1378.

issues; Animal models; Pharmacogenetics; Prenatal diagnosis; Dysmorphology. The manuscript management system is completely online and includes a very quick and fair peer-review system, which is all easy to use. Visit http://www.dovepress.com/testimonials.php to read real quotes from published authors. 